Received: 4 June 2018

Accepted: 4 December 2018

Published online: 30 January 2019
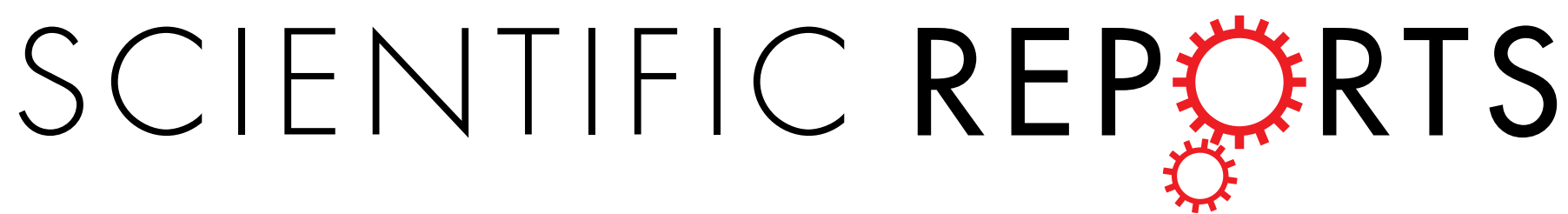

\title{
OPEN
}

\section{Imaginal disc growth factor 4 regulates development and temperature adaptation in Bactrocera dorsalis}

\author{
Xinyue Gu, Zhihong Li, Yun Su, Yan Zhao \& Lijun Liu
}

The oriental fruit fly Bactrocera dorsalis (Hendel) (Diptera: Tephritidae) is an important invasive pest with high reproductive capacity and invasiveness; it has shown remarkable range expansion and brings higher risk to the environment and agriculture. The insect cuticle serves as skin and skeleton, protecting insects against numerous harmful stresses. One gene named imaginal disc growth factor 4 (idgf4) which is involved in cuticle formation, plays an important role in organizing proteins in the chitin-matrix, as well as in adult molting. This gene in the poorly-described glycoside hydrolase 18 (GH 18) family was chosen to study the function of chitinases in insect defense barrier against heat and molting using quantitative real-time PCR (qRT-PCR) and RNA interference (RNAi). qRT- PCR showed that idgf 4 was expressed in all nine developmental stages and was mainly expressed in the early and late pupal, as well as adult stages. Knocking down the idgf 4 gene via RNAi in $3^{\text {rd }}$ instar larvae led to the decreased survival of larvae under high temperatures and malformed individuals as adults. The results indicated the function of the idgf 4 gene in the fruit fly's defense barrier and development. It can provide new insights into understanding the function of one member in the GH 18 family, and may reveal a new potential gene for pest control.

Tephritid flies attack a large variety of fruits, which can be highly priced commodities in many countries ${ }^{1}$. The oriental fruit fly Bactrocera dorsalis is an important invasive Tephritid pest that causes major financial losses in the fruit and horticultural industries because of its high reproductive capacity and invasiveness ${ }^{2-4}$. Over the past 10 years, $B$. dorsalis has shown remarkable range expansion and invaded several new continents ${ }^{5}$. As noted in recent studies, four major pest species, $B$. dorsalis, $B$. philippinensis, B. papayae and $B$. invadens, belong to a $B$. dorsalis complex, which makes it more widely distributed and a higher risk than previously thought ${ }^{6}$. Especially against the background of climate change, the potential distribution of $B$. dorsalis increases significantly in the projected scenarios ${ }^{7}$. Considering its role in high levels of economic loss and an expanding wider distribution, several methods, including destruction of the fallen and infested fruits, cold and heat treatments, bait sprays, biological control methods, sterile insect techniques (SIT) and usage of insecticides have been developed in the last few decades for controlling this notorious pest ${ }^{8-15}$. Among all these methods, insecticides still represent the most effective strategy ${ }^{15,16}$. However, due to the rapid development of insecticide resistance, the control of oriental fruit flies has become difficult ${ }^{7}$. The usefulness of RNA interference (RNAi) in functional genomic research in insects and its considerable potential for the control of pest insects has recently been suggested ${ }^{17}$. Moreover, RNAi can also be used effectively in some classic methods of insect control, such as SIT ${ }^{18,19}$. Using an RNAi approach successfully for pest control mainly relies on effective target gene selection, and it is of great significance to find the potential target genes for the development of this new technology in $B$. dorsalis ${ }^{20}$.

The glycoside hydrolase 18 (GH 18) family of chitinases is a key family in insects that plays various roles in insect cuticle development and molting ${ }^{21-23}$. Based on phylogenetic analyses, the GH 18 family is encoded by a large number of diverse genes and can be classified into five groups ${ }^{24}$. Imaginal disc growth factor (IDGFs), belonging to the group V chitinase, were first identified by fractionating conditioned medium from Drosophila imaginal disc cell cultures ${ }^{25,26}$. Imaginal disc growth factor (IDGFs) were confirmed to be the proteins cooperating with insulin that promote the growth of cell lineages derived from imaginal discs in D. melanogaster ${ }^{25,27,28}$. 
Additionally, this type of cooperation with insulin can be found in other species, such as Mamestra brassicae ${ }^{29}$. In a recent study, the five nonenzymatic idgfs $(i d g f 1,3,4,5,6)$ in Drosophila were certified as structural protein genes to maintain the epithelial apical extracellular matrix (ECM) scaffold against chitinolytic degradation, and also participate in many vital physiological processes of insects such as adult eclosion, development regulation and blood sugar reduction ${ }^{22,30-34}$.

Several studies have focused on the function of individual $i d g$ fgenes or IDGF proteins in different species ${ }^{22,25,31,32,35-38}$. In the functional study of $i d g f$ genes, after the individual $i d g f 1$, idgf 3 , idgf4, idgf5 and idgf6 genes were knocked down via RNAi in Drosophila, the larvae displayed serious epidermal lesions and narrowed ECM thickness, as well as a deformed epidermal chitin-matrix of varying degrees ${ }^{22}$. In addition, starvation was found to cause a decline in the expression level of one idgf gene in the blood of larvae in silkworms, which suggests that this gene may reduce the blood sugar, and thus can be used for screening human hypoglycemic drug ${ }^{32}$. In the functional study, one purified IDGF protein was demonstrated an extremely high level throughout the rapid growth period of organs in Bombyx mori ${ }^{35}$. In vitro cell growth tests showed that, in combination with insulin, recombinant IDGF1 or IDGF2 proteins greatly stimulated the growth of cultured imaginal disk cells in Drosophila ${ }^{25,38}$. Interestingly, there are several factors like the diet and nutrition, which can regulate the secretion of one IDGF in silkworms. A high-glucose diet can suppress insect growth, however, IDGF has been confirmed to be involved in regulating the development of the midgut under this unusual diet condition ${ }^{37}$. Although a large number of studies have generally focused on the function in larval stages, only two related papers were founded about another important developmental stage: pupae. In Tribolium castaneum, dsidgf4 injected into penultimate or last instar larvae resulted in normal pupation but caused death during adult eclosion ${ }^{31}$. In B. mori, proteins with a critically different expression profile between wild type and scaleless wing mutants were verified and revealed that one idgf gene was related to the development and differentiation of scale cells ${ }^{36}$.

The efficient development of the cuticle which is a complex exoskeleton with diverse functions, leads to the evolutionary success of insects. The insect cuticle that formed from structural cuticle proteins and chitin serves both as skin and skeleton, protecting insects against invading pathogens and numerous other harmful stresses ${ }^{22,39}$. Several studies have shown that healthy and dark cuticles are associated with invasive abilities such as temperature adaptation and desiccation resistance ${ }^{40-44}$. In a major destructive insect pest called Ceratitis capitata, in-depth curation of more than 1800 mRNAs showed that specific gene expansions could be related to invasiveness and host adaptation, and gene families of cuticle proteins are important families among these genes ${ }^{40}$. Dark color cuticles in Drosophila are associated with reduced water loss rates ${ }^{41,42}$. D. suzukii 'winter morph' adults with darkened cuticles captured in temperate regions in autumn are the most cold-tolerant form ${ }^{44}$. Meanwhile, the cold tolerance and low temperature activity of winter-acclimated flies is related to increased cuticle melanization ${ }^{43}$.

In summary, previous studies of $i d g f$ genes have paid considerable attention to their function in larval development stages. However, there are few related researches about gene function on stress tolerance or the effect of this gene on pupal development stages. Moreover, studies often focus on the functional study of model species such as Drosophila species and B. mori ${ }^{22,25,32,35-37}$. There are few studies on the GH 18 family in nonmodel species, such as Tephritidae. Besides, from our previous transcriptome analysis (available RNA-seq data at NCBI: SRP093863), we found only idgf4 and idgf6 in the GH 18 family were differentially expressed between two Bactrocera species, $B$. dorsalis and $B$. correcta, in the most sensitive development stage of $3^{\text {rd }}$ instar larvae. $B$. dorsalis showed stronger heat plasticity than $B$. correcta, which idgf4 may play some roles in the adaptation ${ }^{45}$. In summary, this study focused on the idgf4 gene, of which little was known especially in nonmodel organisms such as $B$. dorsalis. The expression pattern with qRT-PCR and gene function of $i d g f 4$ with RNAi on heat tolerance in the larval stage and development in the pupal stage was investigated for the first time in Tephritid fruit fly species, and provided some information on functional study and pest control in other Bactrocera species.

\section{Results}

Cloning and characterization of idgf4. Idgf4 (GenBank accession no. MH 250169) was cloned from $B$. dorsalis. The open reading frame (ORF) was $1320 \mathrm{bp}$ encoding 439 amino acids with a calculated molecular mass of $48.369 \mathrm{kDa}$ and a theoretical isoelectric point (pI) of 7.16. To analyze the phenogenetic relationships of idgf4 between $B$. dorsalis and other species, idgf4 gene sequences from 21 species in Drosophila and Tephritidae (Table S1) were collected by BlastP in GenBank. The conserved domains of idgf4 protein were predicted using NCBI BLAST and the protein contained one GH 18-IDGF domain, one glyco-18 domain (glyco-hydro-18 domain) and one Chi A domain (Fig. 1). Among all the Tephritid fruit flies, we found that there were two types of the genes with three same domains as same as the phylogenetic tree (Figs 1 and 2). Nucleotide sequence analysis revealed that the idgf4 of B. dorsalis had the highest identity with a homolog from B. latifrons (96\%), followed by those from B. oleae (93\%), Zeugodacus cucurbitae (90\%), and C. capitate (90\%). Compared to the similar Drosophila species, the sequence had a highest identity of idgf4 with $D$. kikkawai (79\%). To investigate the evolutionary relationship between idgf4 in $B$. dorsalis and other $i d g f 4$ s, a phylogenetic analysis was performed with two different methods, the neighbor-joining and the maximum-likelihood methods. The phylogenetic tree indicated the relationship between insect idgf4s (Figs 2 and S1). It showed that all $i d g f 4 \mathrm{~s}$ were highly conserved among the similar species. The two methods showed the similar results, in which the idgf4 in B. dorsalis was clustered closed to the other two Bactrocera species (Figs 2 and S1). Interestingly, two species in Tephritidae, five isoforms of idgf4 in Z. cucurbitae as well as two in C. capitata formed two groups. One group in Z. cucurbitae was similar to C. capitata isoform X2, and one was more similar to the Bactrocera group (Figs 2 and S1). Apart from three species in Drosophila, the other species were clustered together and divided into two big groups. The amino acid sequence alignment and evolutionary relationship suggested that idgf4s were highly conserved among other Bactrocera species.

Expression of $i d g f 4$ in nine different development stages of $B$. dorsalis. The expression of idgf4 differed significantly in certain developmental stages (Tukey HSD tests: $\mathrm{P}<0.05$ ). In the larval stage, idgf4 was 
A B. dorsalis MKFVILLALS LAVLASAQHA SNSHLVCYYD GSSYVREGLS KLHTNDLEPA LQFCTHLVYG YASISPTSNK LVSSNDKLDL DIGSGLYRTV TGYKKKYPHL B. latifrons-PREDICTED MKFVILLALS LAVLASAQHA SNSHLVCYYD GSSYVREGLS KLHTNDLEPA LQFCTHLVYG YASISPTSNK LVSSNDKVDL DIGSGLYRTV TGYKKKYPHL B. Oleae-PREDICTED MKFVILLALS LAVLASAQHA SNSHLVCYYD GSSYAREGLS KLHTNDLEPA LQFCTHLIYG YASISPTSNK LVSNNDKLDL DIGSGLYRTV TGYKKKKYPH

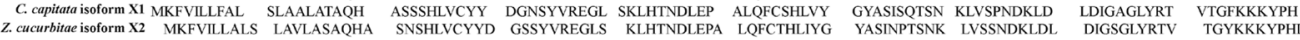
Z. cucurbitae isoform X5 MKFVILLALS LAVLASAQHA SNSHLVCYYD GSSYVREGLS KLHTNDLEPA LQFCTHLIYG YASINPTSNK LVSSNDKLDL DIGSGLYRTV TGYKKKYPHL

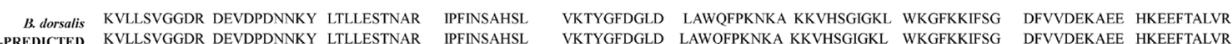
B C. capitata isoform X1 LKVLLSVGGD RDEVDPDNNK YLTLLESANA RIPFINSAHS LVKTYGFDGL DLAWOFPKNK PKKVHSGIGK FWKGFKKIFS GDFVVDEKAE EHKEEFTALV cucurbitae isoform X2 KVLLSVGGDR DEMDPDNNKY LTLLESTNAR RPFINSAHSL VKTYGFDGLD LAWOFPKNKA KKVHGSIGKF WKGFKKIFSG DFVVDEKAEE HKEEFTALVR KVLLSVGGDR DEMDPDNNKY LTLLESTNAR IPFINSAHSL VKTYGFDGLD LAWQFPKNKA KKVHGSIGKF WKGFKKIFSG DFVVDEKAEE HKEEFTALVR

B. dorsalis ELKNAFRPDG YLLGLSVLPN VNSSLFYDVP AIVNNLDYVN LHAYDFOTPE RNPEVADFPA PIYELNERNP EANINFOTQY WLNNHCPATK INVAVAAYGR W

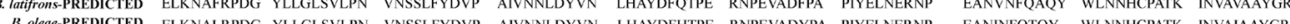
c. oleae-Patata isoform X1 ELKNALRPDG YLLGLSVLPN VNSSLFYDVP AIVNNLDYVN LHAYDFHTPE RNPEVADYPA PIYELNERNP EANINFQTQY WLNNHCPATK INVAIAAYGR z. cucurbitac isoform X2 ELKNAFRPDG YLLGLSVLPN VNSSLFYDVP AIYNNLDYVN LHAYDFOTPE RNPEVADFPA PIYELNERNP EANINFOAOY WLNNHCPATK INVAVAAYGR

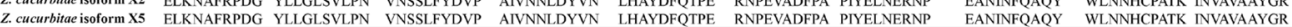

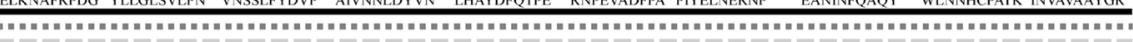

B. dorsalis AWKMTKDSGL TGVPPVVETD GVAPAGTQTQ KPGLLSWPEV CGKLPNPANQ HLKGADGPLR KVGDPTKRFG NYAYRSADDS GENGIWVGYD DPDTAANKAA R

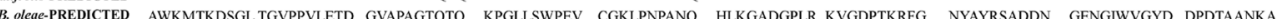
B. olece-PREDCTED AWKMTKDSGL TGVPPVETD GVAPAGTQTQ KPGLLSWPEV CGKLPNPANQ HLKGADGPLR KVGDPTKRFG NYAYRSADDN GENGIWVGYD DPDTAANKAA z. cucurbitae isoform X2 AWKMTKDSGLTGVPPVVVETD GVAPAGTOTO KPGLLSWPEV CGKLPNPANO HLKGADGPLR KVGDPTKRFG NYAYRSADDS GENGIWVGYD DPDTANKA

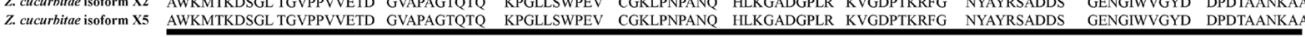

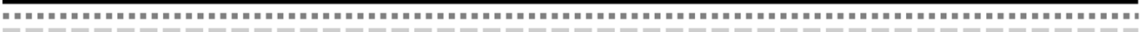

B. dorsalis FVKARGLGGI ALVDLSFDDF RGACTGDKYP ILRAIKFKL

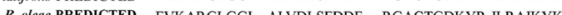

C. capitata isoform X1 AFVKSRGLGG VALVDLSFDD FRGACTGDKY PII RAIKFKL

cucurbita isoform X2 FVKARGLGG

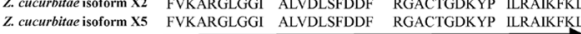

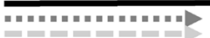

B C. capitata isoform X2 MLKVIYLLTA LCIGSLQATK

MVCYYDSSSQ YREGSAQVSL QDLEPALQFC NYLVYGYAGV DPETYQLRPI NKDLDVGRNH YRTITNLRRN HPQLLLLLSV

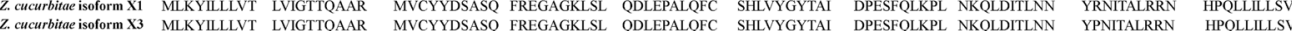

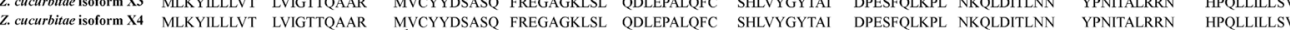
MVCYYDSASQ FREGAGKLSL QDLEPALQFC SHLVYGYTAI DPESFQLKPL NKQLDITLNN YPNITALRRN HPQLLILLSV

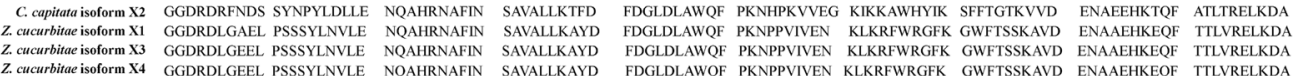
GGDRDLGELL PSSSYLNVLE NQAHRNAFIN SAVALLKAYD FDGLDLAWQF PKNPPVIVEN KLKRFWRGFK GWFTSSKAVD ENAAEHKEQF TTLVRELKDA

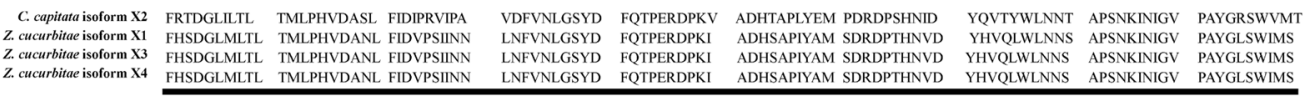
Z. cucurbitac isoform X3 3 FHSDGLMLTL TMLPHVDANL FIDVPSINN LNFVNLGSYD FQTPERDPKI ADHSAPIYAM SDRDPTHNVD YHVQLWLNNS APSNKINIGV PAYGLSWIMS Z. cucurbitae isoform X4 FHSDGLMLTL TMLPHVDANL FIDVPSIINN LNFVNLGSYD FQTPERDPKI ADHSAPIYAM SDRDPTHNVD YHVQLWLNNS APSNKINIGV PAYGLSWIMS

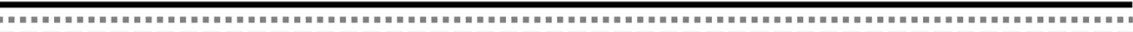

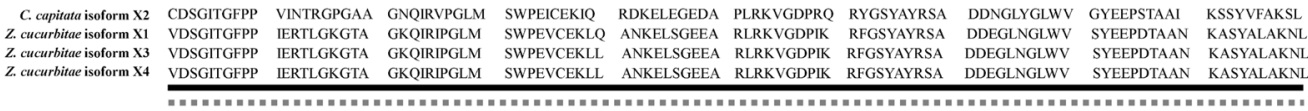

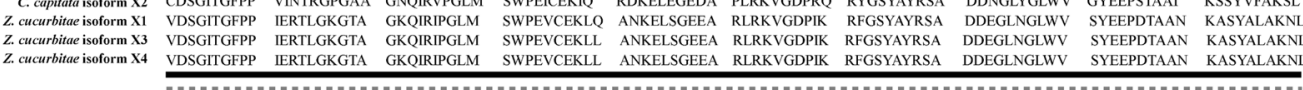
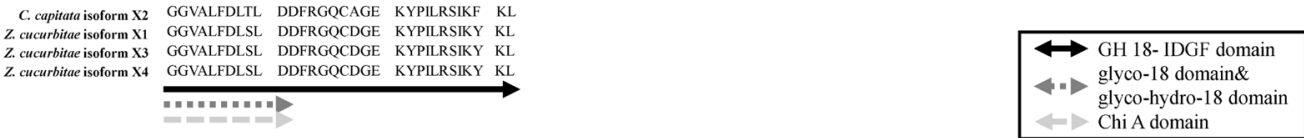

Figure 1. Protein sequence alignments of all the idgf4 proteins in the Tephritid fruit flies based on NCBI BLAST results. (A) The protein sequence alignments using sequences similar to that of $B$. dorsalis. (B) The protein sequence alignments using sequences different from that of $B$. dorsalis. The alignments that present three predicted and conserved domains contained one GH 18- IDGF domain, one glyco-18 domain (glycohydro-18 domain) and one Chi A domain.

expressed in the $1^{\text {st }}$ instar and tended to stabilize until the late third instar. In the pupal stage, the highest level of mRNA expression was detected in early pupae $(\mathrm{P}=0.000)$, then declined during medium pupae and recovered to the second highest level of expression in late pupae $(\mathrm{P}=0.000)$. In adults, the relative expression of $i d g f 4$ on day 10 was significantly higher than that on day $1(\mathrm{P}=0.029)$ and the third highest expression level was measured in the 10-day-old adults. Also, idgf4 expression differ significantly among the other stages, i.e., the expression levels of larval stages (L1, L2, L3-1) compared to L3-3, P-M, and A-E (Fig. 3). The different expression levels indicate that idgf 4 has special physiological roles in different developmental stages. 


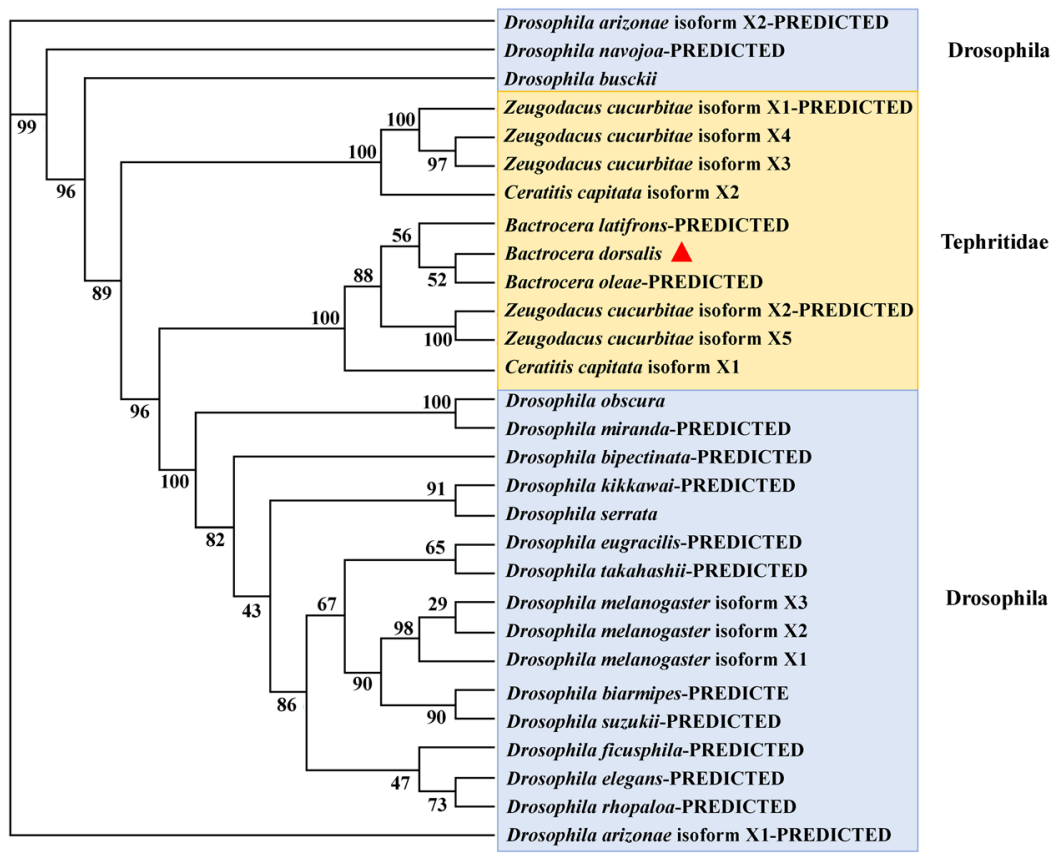

Figure 2. The phylogenetic analysis of $i d g f 4$ using maximum-likelihood method in RAxML. ModelGenerator 0.85 was used to find the optimal amino acid substitution model and the LG $+\mathrm{G}$ model was selected. One thousand Bootstrap iterations were conducted to obtain branch support values. The B. dorsalis idgf4 we got is labeled with a red triangle. The amino acid and nucleotide sequences were downloaded from NCBI. The accession numbers of the genes are designated with the corresponding abbreviations and are listed in Table S1.

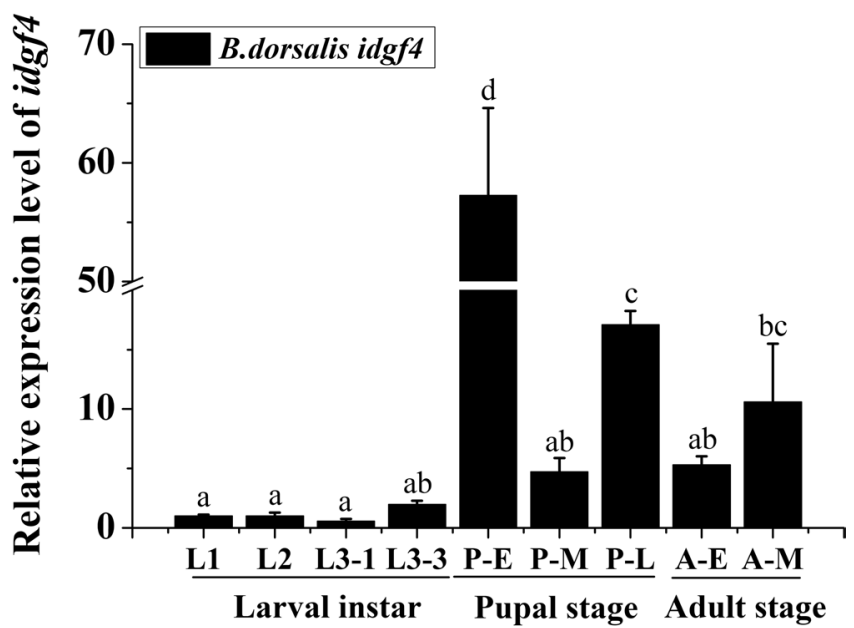

Figure 3. The expression of $i d g f 4$ at nine developmental stages of $B$. dorsalis. The nine developmental stages examined include the $1^{\text {st }}$ instar larvae (L1), $2^{\text {nd }}$ instar larvae (L2), $3^{\text {rd }}$ early-instar larvae (L3-1), $3^{\text {rd }}$ instar larvae (L3-3), 1-3 days mixed pupae as early pupae (P-E), 4-6 days as medium pupae (P-M), and 7-9 days as late pupae (P-L), 1-day adults (A-E) and 10-day adults (A-M). The results are presented as the relative expression after normalization against the endogenous $18 s R N A$ gene. Expression is relative to the gene expression in $1^{\text {st }}$ instar larvae (assigned a value of 1). Different letters above the bars represent significant differences at $P<0.01$, as determined by Tukey's HSD tests.

Silencing of idgf4 caused the low heat tolerance and morphogenesis in B. dorsalis. In the functional study of the larval stage. First, we tested the expression level of $i d g f 4$ under extremely high temperatures without dsidgf 4 feeding. In the extreme high temperature group $\left(45^{\circ} \mathrm{C}\right.$ for 1 hour followed by $4 \mathrm{~h}$ at $\left.25^{\circ} \mathrm{C}\right)$, the idgf4 expression level of insects that survived successfully was 3.15 times higher compared to the control group $\left(25^{\circ} \mathrm{C}\right.$ for $\left.5 \mathrm{~h}\right)$ (t-test: $\mathrm{P}=0.012$, Fig. $\left.4 \mathrm{~A}\right)$. We wondered what would happen if we decreased the expression of idgf4, so RNAi was used to study the response after a decline in idgf4 expression. After 5-day-old $3^{\text {rd }}$ early-instar larvae of $B$. dorsalis were exposured to ds $i d g f 4$ at $1000 \mathrm{ng} / \mu$ concentration for 48 hours, the target gene idgf4 showed $69.2 \%$ and $73.6 \%$ silencing compared to $\mathrm{d} s G F P$-feeding and $\mathrm{H}_{2} \mathrm{O}$-feeding groups, respectively ( $\mathrm{t}$-test: 
A

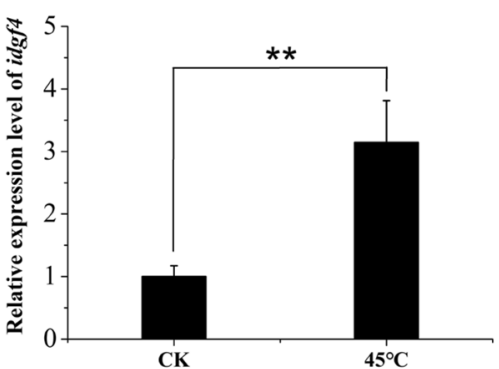

B

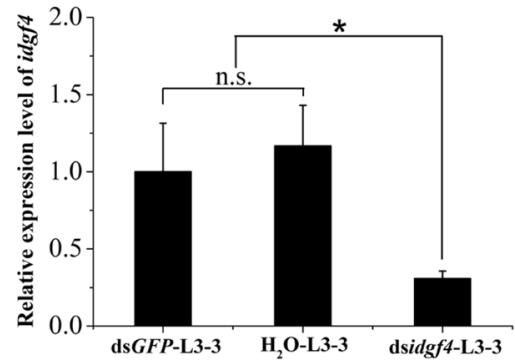

C

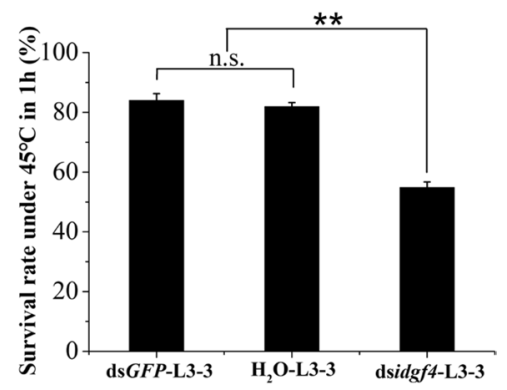

Figure 4. Effect of heat treatment on B. dorsalis larvae. (A) Relative expression level of $i d g f 4$ in $3^{\text {rd }}$ instar larvae (7-day larvae) at $45^{\circ} \mathrm{C}$ for $1 \mathrm{~h}$. (B) The relative expression level of idgf4 after feeding dsidgf4. (C) The survival rate of $B$. dorsalis under high temperature treatment after silencing. All the fruit flies in the function study in $\left(\right.$ B,C) were 5-day-old $3^{\text {rd }}$ early-instar larvae exposed to dsRNA at $1000 \mathrm{ng} / \mu \mathrm{l}$ concentration or $\mathrm{H}_{2} \mathrm{O}$ for $48 \mathrm{~h}$ at $25^{\circ} \mathrm{C}$. ** indicates a statistically significant difference in idgf4 mRNA expression and survival rate between the treatment group and the control ds GFP and $\mathrm{H}_{2} \mathrm{O}$ groups $(P<0.01$, t-test $)$ * indicates a statistically significant difference in idgf4 expression between the dsidgf4 group and the control dsGFP and $\mathrm{H}_{2} \mathrm{O}$ groups $(P<0.05$, t-test). "n.s." indicates no statistically significant difference in idgf4 expression between the control dsGFP and $\mathrm{H}_{2} \mathrm{O}$ groups.

A

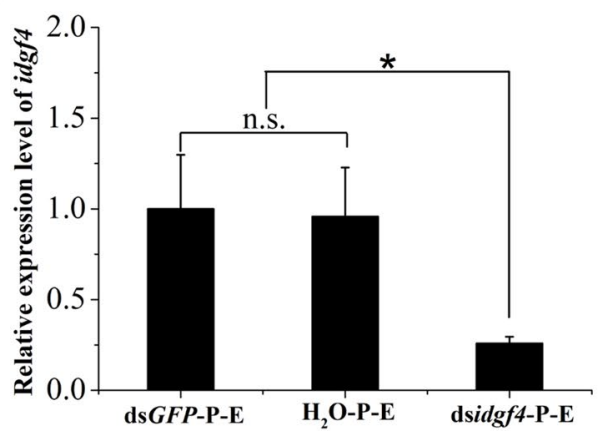

B

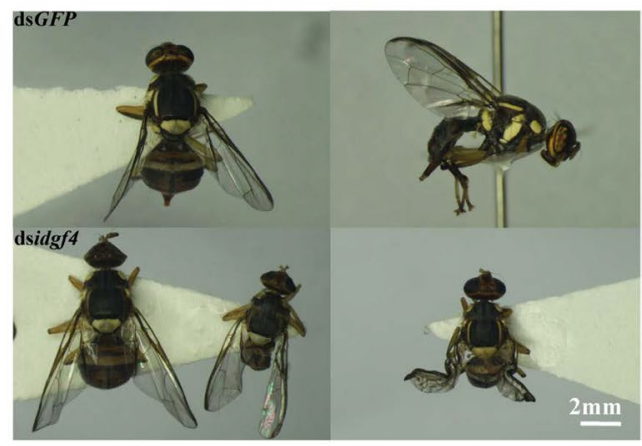

Figure 5. Effect of silencing idgf4 in B. dorsalis. (A) The relative expression level of idgf4 after feeding dsidgf4 in early pupae. (B) Malformed individuals after the silencing. All the fruit flies in the function study in Fig. 5 were 5-day-old $3^{\text {rd }}$ early-instar larvae exposed to dsRNA at $1000 \mathrm{ng} / \mu \mathrm{l}$ concentration or $\mathrm{H}_{2} \mathrm{O}$ for $96 \mathrm{~h}$ at $25^{\circ} \mathrm{C}$. * indicates a statistically significant difference in idgft mRNA expression between the dsidgft group and the control dsGFP and $\mathrm{H}_{2} \mathrm{O}$ groups $(P<0.05$, t-test). "n.s." indicates no statistically significant difference in idgf4 expression between the control dsGFP and $\mathrm{H}_{2} \mathrm{O}$ groups.

$\mathrm{P}=0.047$ and 0.011 , Fig. $4 \mathrm{~B}$ ). Afterwards, the survival rate was detected under the treatments of $45^{\circ} \mathrm{C}$ for 1 hour followed by $4 \mathrm{~h}$ at $25^{\circ} \mathrm{C}$. Compared to the dsGFP-feeding and $\mathrm{H}_{2} \mathrm{O}$-feeding groups, the survival rates decreased by $29.1 \%$ and $27.1 \%$, respectively (Tukey's HSD test: $\mathrm{P}=0.000$, Fig. $4 \mathrm{C}$ ).

In the functional study of the pupal stage. After 5-day-old $3^{\text {rd }}$ early-instar larvae of $B$. dorsalis were exposed to dsidgf4 at $1000 \mathrm{ng} / \mu \mathrm{l}$ concentration for $96 \mathrm{~h}$ at $25^{\circ} \mathrm{C}$, the target gene $i d g f 4$ showed $74.1 \%$ and $73.0 \%$ silencing compared with dsGFP-feeding and $\mathrm{H}_{2} \mathrm{O}$-feeding groups, respectively ( $\mathrm{t}$-test: $\mathrm{P}=0.037$ and 0.036, Fig. $5 \mathrm{~A}$ ), through the expression level detection in the early pupal stage using qRT-PCR. We found approximately $17.5 \%$ of individuals per treatment were deformed after all the fruit flies emerged, exhibiting two types of malformation. One type caused smaller fruit flies, and the other resulted in partly extensible wings, which led to a loss of flight 
capacity (Fig. 5B and Table S3). During subsequent development, approximately 100\% of deformed adults died before sexual maturity, approximately 5 days after emergence. In addition, there were no dead or malformed flies in the control including the $\mathrm{H}_{2} \mathrm{O}$ and dsGFP feeding groups, and all the flies lived for more than half a month.

\section{Discussion}

The poorly described GH 18 family is a key gene family in insect development, with members, such as chitinases, having catalytic activity, as well as some proteins without catalytic activity, such as IDGFs ${ }^{46}$. We have been interested in one gene idgf4 in this family to study its role in insect defense barrier against heat and other key functions during the development in Tephritid fruit fly species for the first time. There are predicted and conserved domains in the idgf4 protein containing the GH 18- IDGF domain, glyco-18 domain (glyco-hydro-18 domain) and Chi A domain (Fig. 1). Two types of the genes with three same domains as same as the phylogenetic tree showed that the genes were highly conserved in the Tephritid fruit flies. However, the idgf4 of two groups are not paralogous, which indicated different phylogenetic relationship between these two types. Besides the overlapping traits, there were no similarity and dissimilarity between two groups. IDGFs have an eight-stranded alpha/beta barrel fold which are concerned with the GH 18 chitinases, but they have a known amino acid substitution to eliminate chitinase catalytic activity ${ }^{25}$. The Chi A domain may indicate it evolved from chitinases and gain new functions as growth factors with the interaction of cell surface glycoproteins ${ }^{25,27}$. Characteristics such as similar arrangement of introns and exons, small size, and different cytological localization make this family an excellent candidate for evolutionary studies ${ }^{28}$. Previous studies, based on the estimated catalytic sites, found idgf family polypeptides with mammalian chitinase-related proteins and confirmed these genes to be homeotic ${ }^{25,47}$. The exon/ intron structures of idgf genes in six forms of Drosophila were also compared ${ }^{28}$. However, these studies did not provide any further details about the members of the idgfgene family or certain genes among insects. Using idgf4 nucleotide sequences of Drosophila and Tephritidae in GenBank, we applied the neighbor-joining method and maximum-likelihood method to obtain a phylogenetic tree. Idgf4 in B. dorsalis has high identity with the homologues in other Tephritid fruit flies (Figs 2 and S1). The highly conserved trait reveals the importance of encoding glycosyl hydrolases among similar species and analogous roles in ECM dynamics across the insect taxa ${ }^{22}$. Idgfs are structurally related to chitinases such as the Chi A domain suggesting that the family of idgfs likely evolved from chitinases ${ }^{25,27}$. However, they acquired a new growth-promoting function, interacting with cell surface glycoproteins implicated in growth-promoting processes.

The idgf4 gene in $B$. dorsalis is continuously expressed from $1^{\text {st }}$ instar larvae through pupae and adults. However, it mainly expresses in the early and late pupal, and mature adult stages. The expression pattern of idgf4 in different developmental stages indicates that it may play distinct roles during insect growth and development. This observation is similar to the previous study in which idgf genes were expressed at rather constant levels throughout larval and pupal development in Drosophila ${ }^{22}$. UAS-RNAi-mediated knockdown of the individual idgf4 gene displayed serious epidermal lesions at wounded sites of $1^{\text {st }}$ instar larvae and the wounded epidermal cuticle barrier lost integrity ${ }^{22}$. Although a large number of studies have paid more attention to its expression in the larval stage, in B. dorsalis, it is mainly expressed in the early and late pupal stages as well as adult stages ${ }^{22,35}$. The knockdown studies in Drosophila identified idgfgenes involved in cuticle molting during the pupal stage, and the gene reduction could cause pupal lethality ${ }^{22}$. In general, we chose pupal stages when the idgf4 gene was expected to play important roles on development as the highest expression levels, to study the function of the idgf4 gene $^{22}$.

The ECM, whose formation involves idgf4, functions as a local defense barrier against a hostile environment; here we studied the gene function of $i d g f 4$ in extreme temperature defense ${ }^{22}$. We used an extremely high temperature to test the gene function in protection of larvae from a hostile environment. The development and growth of insects are greatly affected by environmental temperatures due to their poor ability to adjust and maintain their body temperature as poikilotherms ${ }^{45}$. Among all the stages, larvae were chosen because they have a strong selection for heat resistance and plasticity as they are exposure to thermal stress inside the fruits ${ }^{45}$. The inadequate environment of high temperature leads to an increased expression of $i d g f 4$ in surviving larvae. The increasing expression pattern of this gene might reveal its involvement in heat tolerance. Besides, the comparative transcriptome analyses also indicated $i d g f 4$ may play some roles in the adaptation ${ }^{45}$. We decreased the expression of $i d g f 4$ using RNAi to study its effect on heat acclimation. After the insects were fed with dsidgf4 for $48 \mathrm{~h}$, the expression level of $i d g f 4$ decreased significantly. Meanwhile, the survival rate after the feeding also had a significant drop. In previous research, the idgf4 gene was required for exoskeletal barrier function that led to the normal development of a healthy cuticle throughout larval development ${ }^{22}$. The idgf genes also showed a potential function in insect temperature adaptation in desert beetle Microdera punctipennis, in which low temperatures, such as $4^{\circ} \mathrm{C}$ and $-4^{\circ} \mathrm{C}$ could upregulate the expression of six genes, including idgf2, in the GH 18 family ${ }^{48}$. We showed the same results; that high temperature can upregulate the expression of $i d g f 4$ and that silencing this gene can decrease the survival rate under heat stress in B. dorsalis. Our results also indicated that the expression of $i d g f 4$ can help to increase the heat tolerance (Fig. 4). Although the inside of fruits actually provides insects with a much more stable environment than soil, where pupae develop, the larval stage was still chosen to study the heat influence. In our previous study, the $3^{\text {rd }}$ instar larval stage was found to be the most sensitive stage to heat ${ }^{45,49}$. However, compared to the larvae, pupae could withstand a great range of temperature changes based on the survival rate after different temperature treatments in Bactrocera species ${ }^{50}$.

Some studies certified that the idgf4 gene is related to the growth and development of insects ${ }^{22,25,27,36}$. This gene is involved in chitin-based cuticle development, imaginal disc development and wound healing in $D$. melanogaster according to Flybase (www.flybase.org). The histological analysis of third instar larval individuals, in which idgf4 had been knocked down, showed unusually narrowed ECM thickness and deformed ECMs ${ }^{22}$. In addition, gene function screening via RNAi in T. castaneum showed that the idgf4 gene was required for adult eclosion, but had no effect on adult female fecundity or fertility ${ }^{31}$. In our research, the expression of $i d g f 4$ was highest in the early and late pupal stages, which indicated its possible function for insect growth and development 
during the pupal stage (Fig. 3). Although all the treated individuals emerged after the silencing, approximately $17.5 \%$ of malformed individuals were found in the dsidgf4-feeding group, including smaller and abnormally extensible winged adults that could not survive to sexual maturity (Fig. 5). The imperfect development implied that they had weak diffusion and competition ability which led to the loss of flight ability compared to the healthy flies. In addition, in all the functional studies, two control groups, including irrelevant dsRNA of GFP and $\mathrm{H}_{2} \mathrm{O}$ without any dsRNA, were used, and no difference were found between these two different control groups. No dead or malformed flies in the control groups indicated that the malformed development was related to the idgf4 gene knockdown, not the RNAi methodology itself. The miniaturization of their body size and the evolution of flight and wings, which enhance the ability of insects to colonize novel ecological habitats, profoundly influences all aspects of their biology, from development to behavior ${ }^{51}$. In insects, body size affects important fitness variables such as mate selection, predation and tolerance to heat, cold and starvation ${ }^{52}$. In addition, miniaturization imposes steep demands on the flight system because smaller insects must flap their wings at higher frequencies to generate sufficient aerodynamic forces to stay aloft ${ }^{51}$. During pupation in Drosophila, the development of imaginal discs and the wing is a key developmental period while the insect is in a compacted form ${ }^{53}$. These results also revealed that this gene in $B$. dorsalis had the same function in the development of wings and also the same expression pattern as the one in silkworms, whose mRNA copies of $i d g f$ were less during the early pupal stage in scaleless mutants ${ }^{35}$. These changes in wings might make it impossible for fruit flies to disperse and reproduce.

$B$. dorsalis has shown a remarkable range expansion over the past 10 years and has invaded several new continents ${ }^{5}$. With global warming in recent years, B. dorsalis, poses a significant risk to both agriculture and the environment. Recently, the dsRNA designed to target pest genes has emerged as a promising strategy for improving pest control ${ }^{54}$. In this study, we demonstrated that the expression of the idgf4 gene in B. dorsalis was significantly decreased by feeding insects the dsRNA of $i d g f 4$. Considering its function in the larval and pupal stages, idgf4 can be used as a potential target gene for pest control. Although we reduced the expression of this gene in early pupal period, nearly $20 \%$ of $B$. dorsalis were unable to recover, even after 10 days of pupation in our study. The irreparability of wings and body size, as well as the highly conserved traits, indicate that idgfs are key genes for pest.

Despite the economic importance of $B$. dorsalis, only limited information regarding the molecular and developmental biology of this insect exists ${ }^{55}$. Here, we report the expression pattern and function of one gene in a poorly described idgf family in depth. It is widely accepted that strong heat tolerance and healthy growth contribute to further invasion ability ${ }^{45}$. After the $i d g f 4$ knockdown experiment, the decreased survival ability under high temperature in larvae and malformed individuals in pupae indicated the function of this gene in invasive fruit fly development and heat tolerance. Additionally, our results provide new insights into the function of $i d g f$ family members and may reveal a new potential gene for pest control.

\section{Methods}

Insects. Individuals of $B$. dorsalis were collected from their original invaded province of Guangdong and reared as described by Yuan et al. ${ }^{56,57}$. All the fruit flies were maintained at $25^{\circ} \mathrm{C}$, with a $14 \mathrm{~h} \mathrm{light:} 10 \mathrm{~h}$ dark photoperiod. Eggs and larvae were given an artificial diet consisting of $120 \mathrm{~g}$ sugar, $40 \mathrm{~g}$ yeast extract, $10 \mathrm{~g}$ peptone, $10 \mathrm{~g}$ agar, $8.8 \mathrm{~g}$ antibiotic and $1000 \mathrm{ml} \mathrm{H}_{2} \mathrm{O}$. The adult flies were cultured using $25 \%$ sucrose and $75 \%$ peptone. The fruit flies had been maintained in the laboratory for approximately 10 generations prior to the experiments to eliminate temperature plastic traits derived from local environmental influence. Two hundred individuals were cultured in a $45 \mathrm{~cm} * 45 \mathrm{~cm} * 50 \mathrm{~cm}$ insect rearing cage and three cages totally were used to culture the fruit flies. We also put 10-50 new field fruit flies from Guangzhou to each cage every half year.

RNA extraction and cDNA synthesis. Each stage had five replicates for idgf4 expression pattern detection and every replicate with different number of individuals (per replication: L1 50 larvae, L2 40 larvae, L3-1 and L3-3 30 larvae, pupal stage including P-E, P-M and P-L 6 pupae, adult stages including A-E and A-M 10 adults) were collected randomly and mixed together. We distinguish each stage based on the body size and the development of oral hooks through microscope according to methods provide by Zhou's study ${ }^{58}$. The mouth hook length and width for the $1^{\text {st }}(0.0846 \pm 0.0005 \mathrm{~mm}, 0.0116 \pm 0.0003 \mathrm{~mm}), 2^{\text {nd }}(0.1512 \pm 0.0013 \mathrm{~mm}, 0.0308 \pm 0.0007 \mathrm{~mm})$ and $3^{\text {rd }}$ instars $(0.2913 \pm 0.0015 \mathrm{~mm}, 0.0677 \pm 0.0013 \mathrm{~mm})$ were distinct, respectively. The average body length of the $1^{\text {st }}, 2^{\text {nd }}$ and $3^{\text {rd }}$ instar larvae were $0.9725 \pm 0.0275 \mathrm{~mm}, 3.2011 \pm 0.0961 \mathrm{~mm}$ and $7.9726 \pm 0.2006 \mathrm{~mm}$. The average body width of the $1^{\text {st }}, 2^{\text {nd }}$ and $3^{\text {rd }}$ instar larvae were $0.2111 \pm 0.0049 \mathrm{~mm}, 0.5882 \pm 0.0181 \mathrm{~mm}$ and $1.4113 \pm 0.0309 \mathrm{~mm}$. Based on the body size and oral hooks, we can know the exact developing time for each larvae stage under our culturing condition ${ }^{58}$. RNAi efficiency was detected with five replicates in the larval stage and four replicates in the pupal study, and one replicate containing 10 randomly collected larvae or pupae and multiple insects together for sampling. RNA was extracted from the whole body of larvae, pupae and adults using the RNAsimple Total RNA Kit (Tiangen, China). cDNA was synthesized from 1000 ng total RNA using PrimeScript ${ }^{@}$ RT reagent Kit with gDNA Eraser (Perfect Real Time) (Takara, Japan) following the manufacturer's instructions.

ORF cloning of $\boldsymbol{B}$. dorsalis idgf4. To verify the ORF of $i d g f 4$ in $B$. dorsalis, three pairs of primers were designed based on the conserved regions of the idgf4 from B. oleae, C. capitata, D. melanogaster (sequence from GeneBank) and the sequence of idgf4 from the result of the B. dorsalis transcriptome (No. SRP093863). The primers for cloning and amplification conditions are shown in Tables 1 and S2, respectively. The ORF and conserved domain were identified with the ORF Finder software (http://www.ncbi.nlm.nih.gov/gorf/gorf.html) and NCBI BLAST results (http://blast.ncbi.nlm.nih.gov/Blast.cgi). The isoelectric point and molecular weight were predicted using the SWISS-MODEL (https://web.expasy.org/compute_pi/). 


\begin{tabular}{|c|c|c|c|}
\hline Gene & Primer & Sequence & Size (bp) \\
\hline \multirow{2}{*}{$B d$ 18s rRNA-rt } & $18 s-r t-F$ & GCGAGAGGTGAAATTCTTGG & \multirow{2}{*}{160} \\
\hline & $18 s-r t-R$ & CGGGTAAGCGACTGAGAGAG & \\
\hline \multirow{2}{*}{$B d E F \alpha 1-r t$} & $E F \alpha 1-r t-F$ & CGTTGGTGTCAACAAGATGG & \multirow{2}{*}{230} \\
\hline & $E F \alpha 1-r t-R$ & TGCCTTCAGCATTACCTTCC & \\
\hline \multirow{2}{*}{$B d G A P D H-r t$} & GAPDH -rt-F & GACGCCTACAAGCCTGACAT & \multirow{2}{*}{221} \\
\hline & GAPDH -rt-R & GTTGAAGCGGGAATGATGTT & \\
\hline \multirow{2}{*}{$B d$ RPL13-rt } & RPL13-rt-F & CAGTTGTACGTTGCGAGGAAT & \multirow{2}{*}{134} \\
\hline & $R P L 13-r t-R$ & TCTTGATGGAGCACGGGAG & \\
\hline \multirow{2}{*}{ Bd idgf4-rt } & idgf4-rt-F & ATGTTCCTCCGCCTTCT & \multirow{2}{*}{152} \\
\hline & idgf4-rt-R & CGCATACCGTTCATAAATAG & \\
\hline \multirow{2}{*}{ Bd cloneidgf4-1 } & idgf4idgf4-whole seq-F-1 & GAAATTTGTCATTTTGCTAGC & \multirow{2}{*}{1300} \\
\hline & idgf4-whole seq-R-1 & GGCACGTAAGATGGGATA & \\
\hline \multirow{2}{*}{ Bd cloneidgf4-2 } & idgf4-whole seq-F-2 & TTACTTTGGAGGCGAGTAGTTC & \multirow{2}{*}{341} \\
\hline & idgf4-whole seq-R-2 & CATTGCTGCTGACAAGTTTATT & \\
\hline \multirow{2}{*}{ Bd cloneidgf4-3 } & idgf4-whole seq-F-3 & TTGGGTGGGCTATGACGA & \multirow{2}{*}{265} \\
\hline & idgf4-whole seq- $R-3$ & ATGCTCCGAACAATGAAACT & \\
\hline \multirow{2}{*}{$B d$ dsidgf 4} & idgf4-dsRNA-F & CATAGCGGCATCGGTAAAT & \multirow{2}{*}{574} \\
\hline & idgf4-dsRNA-R & GAACCATCAGCGCCTTCA & \\
\hline
\end{tabular}

Table 1. Primers used for cloning and real time qRT-PCR amplification.

Phylogenetic analysis. The integrity of homologous amino acid sequences of other species was retrieved from the NCBI server. Sequences were first aligned by the conserved sequences and then phylogenetic analysis was performed using two methods: the neighbor-joining method in the Molecular Evolutionary Genetics Analysis software (MEGA version 5.1) and the maximum-likelihood method in the RAxML analysis ${ }^{59}$. ModelGenerator 0.85 was used to find the optimal amino acid substitution model and the LG $+\mathrm{G}$ model was selected ${ }^{60}$. One thousand bootstrap iterations were conducted to obtain branch support values. All the positions that contained gaps and missing data were eliminated before alignment and phylogenetic analysis.

qRT-PCR detection of gene expression. $\quad$ qRT-PCR was performed using SYBR ${ }^{\circledR}$ Premix Ex Taq ${ }^{\mathrm{TM}}$ II (Tli RNaseH Plus) (Takara, Japan) on an ABI 7500 instrument (USA). All RNA samples were analyzed in triplicate (tech reps). The reactions included $1 \mu \mathrm{cDNA}, 12.5 \mu \mathrm{l}$ SYBR Green mix, $1 \mu \mathrm{l}$ each of forward and reverse prim-

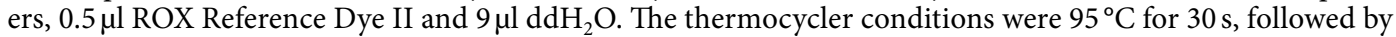
40 cycles at $95^{\circ} \mathrm{C}$ for $5 \mathrm{~s}$ and $52^{\circ} \mathrm{C}$ for $34 \mathrm{~s}$. Melting curve analysis was performed the end of each expression analysis, using the following conditions: $95^{\circ} \mathrm{C}$ for $15 \mathrm{~s}$, followed by $52^{\circ} \mathrm{C}$ for $60 \mathrm{~s}$. Four genes including $18 \mathrm{~s} r R A$, $R P L 13, E F \alpha 1$ and GAPDH were tested the stability to be used as endogenous reference genes by geNorm and $18 \mathrm{~s}$

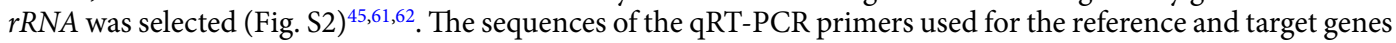
are described in Table 1.

Expression pattern of idgf4. To determine the constitutive expression of $i d g f 4$, fruit flies were collected at the following nine specific developmental stages: 2 -day-old $1^{\text {st }}$ instar larvae, 4 -day-old $2^{\text {nd }}$ instar larvae, 5 -day-old $3^{\text {rd }}$ early-instar larvae, 7-day-old $3^{\text {rd }}$ instar larvae, 1-3 days mixed pupae (early pupae), 4-6 days (medium pupae), 7-9 days (late pupae), 1-day adults (early adults), and 10-day adults (late adults). Every replicate with different number of individuals (see RNA extraction and cDNA synthesis) were collected randomly and mixed together for idgf4 expression pattern detecting. Meanwhile, the 5-day-old $3^{\text {rd }}$-early instar larvae of $B$. dorsalis were collected for the dsRNA feeding experiment. After RNA extraction and cDNA synthesis, the expression pattern was detected using qRT-PCR. Each stage had five replicates.

dsRNA preparation for gene function study. Double-stranded RNA of idgf4 (dsidgf4) was used to knock down idgf4 expression, and double-stranded RNA of green fluorescent protein (dsGFP) and $\mathrm{H}_{2} \mathrm{O}$ without any dsRNA were used as the negative controls. The dsRNAs were synthesized by using the T7 RiboMAX Express RNAi system (Promega, USA). The primers for dsRNA synthesis and amplification conditions are shown in Tables 1 and S2, respectively. The 5-day-old $3^{\text {rd }}$-early instar larvae of $B$. dorsalis were collected and moved into a $50 \mathrm{ml}$ tube with 3 holes on the lid. In the functional study of the larval stage, five replicates were performed for each treatment and each replicate contained 40 larvae. Three grams of artificial diet material with $30 \mu \mathrm{l}$ of a dsRNA solution or $\mathrm{H}_{2} \mathrm{O}$ was used for feeding for $48 \mathrm{~h}$. In the functional study of the pupal stage, four replications were performed for each treatment and each replication contained 30 larvae. Three grams of artificial diet material with $30 \mu \mathrm{l}$ of a dsRNA solution or $\mathrm{H}_{2} \mathrm{O}$ was used for feeding for $96 \mathrm{~h}$. The concentration of the dsRNA solution for the primary exposure was $1000 \mathrm{ng} / \mu \mathrm{l}$.

Heat treatments. To study the effect of idgf4 expression on the temperature tolerance of insects, 30 individuals (7-day-old $3^{\text {rd }}$ instar larvae after hatching) were selected for the temperature treatments without dsRNA feeding. Larvae were exposed to $45^{\circ} \mathrm{C}$, which was selected as the thermal stress endpoint, for $1 \mathrm{~h}$ and experienced 
mortality in the range of $40-50 \%{ }^{45}$. After exposure to the heat stress, the larvae were returned to $25^{\circ} \mathrm{C}$, and their survival rate was scored after $4 \mathrm{~h}$ to prevent insects from heat-shock fainting. A larva was considered dead if no movement was detected when being poked gently by forceps. The survived insects were immediately killed using liquid nitrogen, and stored at $-80^{\circ} \mathrm{C}$ for further molecular analysis. Then, for the $\mathrm{H}_{2} \mathrm{O}$ and dsRNA-feeding larval temperature study, forty larvae (5-day-old $3^{\text {rd }}$ early-instar larvae) were fed an artificial diet supplemented with $\mathrm{H}_{2} \mathrm{O}$ and dsRNA for $48 \mathrm{~h}$. Ten larvae were randomly selected and immersed in the liquid nitrogen to kill for RNA isolation. The remaining 30 individuals (7-day-old $3^{\text {rd }}$ instar larvae: after feeding the dsGFP, $\mathrm{H}_{2} \mathrm{O}$ and dsidgf4 for $48 \mathrm{~h}$, all 5-day-old $3^{\text {rd }}$ early-instar larvae come 7 -day-old $3^{\text {rd }}$ instar larvae) were chosen for the temperature treatments as mentioned before. All larvae were exposed to $45^{\circ} \mathrm{C}$ for $1 \mathrm{~h}$. After exposure to the heat stress, the larvae were returned to $25^{\circ} \mathrm{C}$ and their survival rate was scored after $4 \mathrm{~h}$. The larva was considered dead using the method mentioned before. During the thermal treatments, samples were enclosed in $2 \mathrm{ml}$ tubes with a hole on the lid, were topped with $4 \mathrm{~g}$ diet and incubated in a water bath set to the desired temperature using a PolyScience Programmable Temperature Controller (USA). Each treatment (dsGFP, $\mathrm{H}_{2} \mathrm{O}$ and dsidgf4) included five biological replicates.

Silencing idgf4 in B. dorsalis. For the adult malformation study, thirty 5-day-old $3^{\text {rd }}$ early-instar larvae were fed with dsGFP, $\mathrm{H}_{2} \mathrm{O}$ and dsidgf4 for $48 \mathrm{~h}$ and transferred to a new artificial diet with the same treatment for another $48 \mathrm{~h}$. After $96 \mathrm{~h}$, the larvae developed to maturity and were then transferred into soil for pupation. After one to three days of pupation, ten early pupae were killed for the RNAi efficiency detection. The remaining twenty individuals were continued to be fed as adults and were used for phenotype observation on the $2^{\text {nd }}$ day after emergence. The mortality of emerged individuals was recorded in twenty days after the flies emerged. Each treatment (dsGFP, $\mathrm{H}_{2} \mathrm{O}$ and dsidgf4) included four biological replicates.

Statistical analysis. The qRT-PCR data were analyzed using the $2-\Delta C T$ method ${ }^{63}$. The expression of $i d g f 4$ was quantified in the larvae treated with dsGFP, $\mathrm{H}_{2} \mathrm{O}$ and dsidgf4. Biological replicates were used for statistical analysis. All results from experimental replicates were analyzed using Student's t-test or a Turkey's test using SPSS 20 (IBM Corporation, USA).

\section{Data Availability}

All data generated or analyzed during this study are included in this published article (and its supplementary information file).

\section{References}

1. Vontas, J. et al. Insecticide resistance in Tephritid flies. Pesticide biochemistry and physiology 100, 199-205 (2011).

2. Benelli, G. et al. Sexual communication and related behaviours in Tephritidae: current knowledge and potential applications for Integrated Pest Management. Journal of Pest Science 87, 385-405 (2014).

3. Shi, Y. et al. Ecdysis Triggering Hormone Signaling (ETH/ETHR-A) is required for the larva-larva ecdysis in Bactrocera dorsalis (Diptera: Tephritidae). Frontiers in Physiology 8, 587 (2017).

4. Clarke, A. R. et al. Invasive phytophagous pests arising through a recent tropical evolutionary radiation: the Bactrocera dorsalis complex of fruit flies. Annu. Rev. Entomol. 50, 293-319 (2005).

5. Pieterse, W., Terblanche, J. S. \& Addison, P. Do thermal tolerances and rapid thermal responses contribute to the invasion potential of Bactrocera dorsalis (Diptera: Tephritidae)? Journal of insect physiology 98, 1-6 (2017).

6. Schutze, M. K. et al. Synonymization of key pest species within the Bactrocera dorsalis species complex (Diptera: Tephritidae): taxonomic changes based on a review of 20 years of integrative morphological, molecular, cytogenetic, behavioural and chemoecological data. Systematic Entomology 40, 456-471 (2015).

7. Stephens, A., Kriticos, D. \& Leriche, A. The current and future potential geographical distribution of the oriental fruit fly, Bactrocera dorsalis (Diptera: Tephritidae). Bulletin of Entomological Research 97, 369-378 (2007).

8. Liquido, N. J. Reduction of Oriental Fruit Fly (Diptera: Tephritidae) populations in papaya orchards by field sanitation. J. Agric. Entomol 10, 163-170 (1993).

9. Burikam, I., Sarnthoy, O., Charernsom, K., Kanno, T. \& Homma, H. Cold temperature treatment for mangosteens infested with the oriental fruit fly (Diptera: Tephritidae). Journal of economic entomology 85, 2298-2301 (1992).

10. Armstrong, J. W., Hu, B. K. \& Brown, S. A. Single-temperature forced hot-air quarantine treatment to control fruit flies (Diptera: Tephritidae) in papaya. Journal of economic entomology 88, 678-682 (1995).

11. Roessler, Y. Insecticidal bait and cover sprays. World crop pests 3, 329-337 (1989).

12. Wharton, R. Classical biological control of fruit-infesting Tephritidae. Fruit flies: their biology, natural enemies and control 3, 303-313 (1989).

13. Dyck, V. A., Hendrichs, J. \& Robinson, A. S. Sterile insect technique: principles and practice in area-wide integrated pest management. New Agriculturist 4 (2005)

14. Shelly, T. E., Edu, J. \& McInnis, D. Pre-release consumption of methyl eugenol increases the mating competitiveness of sterile males of the oriental fruit fly, Bactrocera dorsalis, in large field enclosures. Journal of Insect Science 10, 1-16 (2010).

15. Bhagat, D., Samanta, S. K. \& Bhattacharya, S. Efficient management of fruit pests by pheromone nanogels. Scientific reports 3, 1294 (2013).

16. Jin, T., Zeng, L., Lin, Y. Y., Lu, Y. Y. \& Liang, G. W. Insecticide resistance of the oriental fruit fly, Bactrocera dorsalis (Hendel) (Diptera: Tephritidae), in mainland China. Pest management science 67, 370-376 (2011).

17. Huvenne, H. \& Smagghe, G. Mechanisms of dsRNA uptake in insects and potential of RNAi for pest control: a review. Journal of insect physiology 56, 227-235 (2010).

18. Knipling, E. Possibilities of insect control or eradication through the use of sexually sterile males. Journal of Economic Entomology 48, 459-462 (1955).

19. Whyard, S. et al. Silencing the buzz: a new approach to population suppression of mosquitoes by feeding larvae double-stranded RNAs. Parasites \& vectors 8,96 (2015).

20. Wang, Y. B., Zhang, H., Li, H. C. \& Miao, X. X. Second-generation sequencing supply an effective way to screen RNAi targets in large scale for potential application in pest insect control. PloS one 6, e18644 (2011).

21. Huang, Q. S. et al. The GH 18 family of chitinases: their domain architectures, functions and evolutions. Glycobiology 22, 23-34 (2011).

22. Pesch, Y. Y., Riedel, D., Patil, K. R., Loch, G. \& Behr, M. Chitinases and Imaginal disc growth factors organize the extracellular matrix formation at barrier tissues in insects. Scientific reports 6, 18340 (2016). 
23. Pesch, Y. Y., Riedel, D. \& Behr, M. Drosophila Chitinase 2 is expressed in chitin producing organs for cuticle formation. Arthropod structure \& development 46, 4-12 (2017).

24. Genta, F. A. et al. Purification, characterization and molecular cloning of the major chitinase from Tenebrio molitor larval midgut. Insect biochemistry and molecular biology 36, 789-800 (2006).

25. Kawamura, K., Shibata, T., Saget, O., Peel, D. \& Bryant, P. J. A new family of growth factors produced by the fat body and active on Drosophila imaginal disc cells. Development 126, 211-219 (1999).

26. Zhu, Q. S. et al. Domain organization and phylogenetic analysis of the chitinase-like family of proteins in three species of insects. Insect biochemistry and molecular biology 38, 452-466 (2008).

27. Varela, P. F., Llera, A. S., Mariuzza, R. A. \& Tormo, J. Crystal structure of imaginal disc growth factor-2- A member of a new family of growth-promoting glycoproteins from Drosophila melanogaster. Journal of Biological Chemistry 277, 13229-13236 (2002).

28. Ayala, F. J. Polymorphism patterns in two tightly linked developmental genes, Idgf1 and Idgf3, of Drosophila melanogaster. Genetics 162, 177-188 (2002).

29. Zhang, J., Iwai, S., Tsugehara, T. \& Takeda, M. MbIDGF, a novel member of the imaginal disc growth factor family in Mamestra brassicae, stimulates cell proliferation in two lepidopteran cell lines without insulin. Insect biochemistry and molecular biology 36, 536-546 (2006).

30. Galko, M. J. \& Krasnow, M. A. Cellular and genetic analysis of wound healing in Drosophila larvae. PLoS biology 2, e239 (2004).

31. Zhu, Q., Arakane, Y., Beeman, R. W., Kramer, K. J. \& Muthukrishnan, S. Functional specialization among insect chitinase family genes revealed by RNA interference. Proceedings of the National Academy of Sciences 105, 6650-6655 (2008).

32. Li, Y. J. et al. The effect of calorie restriction on growth and development in silkworm, Bombyx mori. Archives of insect biochemistry and physiology 71, 159-172 (2009).

33. Turner, J. R. Intestinal mucosal barrier function in health and disease. Nature Reviews Immunology 9, 799 (2009).

34. Zhang, J. et al. Comparative genomic analysis of chitinase and chitinase-like genes in the African malaria mosquito (Anopheles gambiae). PloS one 6, e19899 (2011).

35. Wang, H. B. et al. Purification and expression analysis of imaginal disc growth factor in the silkworm, Bombyx mori. Journal of insect physiology 55, 1065-1071 (2009).

36. Shi, X. F. et al. Proteomic analysis of the phenotype of the scaleless wings mutant in the silkworm, Bombyx mori. Journal of proteomics 78, 15-25 (2013).

37. Feng, F. et al. Comparative proteomic analysis reveals the suppressive effects of dietary high glucose on the midgut growth of silkworm. Journal of proteomics 108, 124-132 (2014).

38. Hipfner, D. R. \& Cohen, S. M. New growth factors for imaginal discs. Bioessays 21, 718-720 (1999)

39. Noh, M. Y., Muthukrishnan, S., Kramer, K. J. \& Arakane, Y. Cuticle formation and pigmentation in beetles. Current opinion in insect science 17, 1-9 (2016)

40. Papanicolaou, A. et al. The whole genome sequence of the Mediterranean fruit fly, Ceratitis capitata (Wiedemann), reveals insights into the biology and adaptive evolution of a highly invasive pest species. Genome biology 17, 192 (2016).

41. Parkash, R., Aggarwal, D. D., Lambhod, C. \& Singh, D. Divergence of water balance mechanisms and acclimation potential in body color morphs of Drosophila ananassae. Journal of Experimental Zoology Part A: Ecological Genetics and Physiology 321, 13-27 (2014).

42. Ramniwas, S., Kajla, B., Dev, K. \& Parkash, R. Direct and correlated responses to laboratory selection for body melanisation in D. melanogaster: support for melanism-desiccation resistance hypothesis. Journal of Experimental Biology, jeb. 076166 (2012).

43. Toxopeus, J., Jakobs, R., Ferguson, L. V., Gariepy, T. D. \& Sinclair, B. J. Reproductive arrest and stress resistance in winter-acclimated Drosophila suzukii. Journal of insect physiology 89, 37-51 (2016).

44. Stephens, A., Asplen, M., Hutchison, W. \& Venette, R. C. Cold hardiness of winter-acclimated Drosophila suzukii (Diptera: Drosophilidae) adults. Environmental entomology 44, 1619-1626 (2015).

45. Hu, J. T., Chen, B. \& Li, Z. H. Thermal plasticity is related to the hardening response of heat shock protein expression in two Bactrocera fruit flies. Journal of insect physiology 67, 105-113 (2014).

46. Funkhouser, J. D. \& Aronson, N. N. Chitinase family GH 18: evolutionary insights from the genomic history of a diverse protein family. BMC evolutionary biology 7, 96 (2007).

47. Kirkpatrick, R. B., Matico, R. E., McNulty, D. E., Strickler, J. E. \& Rosenberg, M. An abundantly secreted glycoprotein from Drosophila melanogaster is related to mammalian secretory proteins produced in rheumatoid tissues and by activated macrophages. Gene 153, 147-154 (1995).

48. Lu, X. Y., Li, J. Q., Liu, X. N., Li, X. B. \& Ma, J. Characterization and expression analysis of six chitinase genes from the desert beetle Microdera punctipennis in response to low temperature. Cryoletters 35, 438-448 (2014).

49. Jang, E. B. Thermal death kinetics and heat tolerance in early and late third instars of the oriental fruit fly (Diptera: Tephritidae). Journal of economic entomology 84, 1298-1303 (1991).

50. Liu, X. \& Ye, H. Effect of temperature on development and survival of Bactrocera correcta (Diptera: Tephritidae). Scientific Research and Essay 4, 467-472 (2009).

51. Deora, T., Singh, A. K. \& Sane, S. P. Biomechanical basis of wing and haltere coordination in flies. Proceedings of the National Academy of Sciences 112, 1481-1486 (2015).

52. Edgar, B. A. How flies get their size: genetics meets physiology. Nature Reviews Genetics 7, 907 (2006).

53. Turner, C. M. \& Adler, P. N. Morphogenesis of Drosophila pupal wings in vitro. Mechanisms of development 52, 247-255 (1995).

54. Chen, A. E., Zheng, W. W., Zheng, W. P. \& Zhang, H. Y. The effects of RNA interference targeting Bactrocera dorsalis ds-Bdrpl19 on the gene expression of rpl19 in non-target insects. Ecotoxicology 24, 595-603 (2015).

55. Suksuwan, W., Cai, X. L., Ngernsiri, L. \& Baumgartner, S. Segmentation gene expression patterns in Bactrocera dorsalis and related insects: regulation and shape of blastoderm and larval cuticle. The International journal of developmental biology 61, 439-450 (2017).

56. Li, Y. L., Wu, Y., Chen, H., Wu, J. J. \& Li, Z. H. Population structure and colonization of Bactrocera dorsalis (Diptera: Tephritidae) in China, inferred from mtDNA COI sequences. Journal of applied entomology 136, 241-251 (2012).

57. Yuan, S. et al. Introduction to two kinds of artificial diets for mass rearing of adult Bactrocera dorsalis (Hendel). Journal of Huazhong Agricultural University 25, 371-374 (2006).

58. Zhou, X. S., Li, B. S., Liu, B., Wang, Y. J. \& Ye, B. H. Preliminary investigation on the identification of the larval stages of the Bactrocera dorsalis Hendel. Plant quarantine 31, 17-22 (2017).

59. Stamatakis, A. RAxML version 8: a tool for phylogenetic analysis and post-analysis of large phylogenies. Bioinformatics 30 , 1312-1313 (2014).

60. Keane, T. M., Naughton, T. J. \& McInerney, J. O. ModelGenerator: amino acid and nucleotide substitution model selection. National University of Ireland, Maynooth, Ireland 34 (2004).

61. Vandesompele, J. et al. Accurate normalization of real-time quantitative RT-PCR data by geometric averaging of multiple internal control genes. Genome Biology 3, research0034.1 (2002).

62. Shen, G. M., Huang, Y., Jiang, X. Z., Dou, W., \& Wang, J. J. Effect of $\beta$-cypermetherin exposure on the stability of nine housekeeping genes in Bactrocera dorsalis (Diptera: Tephritidae). Florida Entomologist 96, 442-450 (2013).

63. Chen, B. \& Wagner, A. Hsp90 is important for fecundity, longevity, and buffering of cryptic deleterious variation in wild fly populations. BMC evolutionary biology 12, 25 (2012) 


\section{Acknowledgements}

This work was financially supported by the Beijing Nature Science Foundation (6174043) and Basic Scientific Research Operating Expenses of Central Universities (2018QC022). We are grateful to Shaokun Guo from the China Agricultural University for help with providing the sequence information and analysis results in transcriptome, Shiqian Feng from the China Agricultural University for help with providing the methods of phylogenetic analysis, and Guoping Zhan and Chen Ma from the Chinese Academy of Inspection and Quarantine for help with providing insects throughout this work.

\section{Author Contributions}

Xinyue $\mathrm{Gu}$, Zhihong Li and Lijun Liu conducted experiments and results analysis, Xinyue Gu, Yun Su, and Yan Zhao prepared the figures, Xingyue Gu, Zhihong Li and Lijun Liu wrote the manuscript. All authors reviewed the manuscript.

\section{Additional Information}

Supplementary information accompanies this paper at https://doi.org/10.1038/s41598-018-37414-9.

Competing Interests: Dr. Liu and Xinyue Gu declare that the authors have no competing interests as defined by Nature Research, or other interests that might be perceived to influence the results and/or discussion reported in this paper.

Publisher's note: Springer Nature remains neutral with regard to jurisdictional claims in published maps and institutional affiliations.

(c) (i) Open Access This article is licensed under a Creative Commons Attribution 4.0 International License, which permits use, sharing, adaptation, distribution and reproduction in any medium or format, as long as you give appropriate credit to the original author(s) and the source, provide a link to the Creative Commons license, and indicate if changes were made. The images or other third party material in this article are included in the article's Creative Commons license, unless indicated otherwise in a credit line to the material. If material is not included in the article's Creative Commons license and your intended use is not permitted by statutory regulation or exceeds the permitted use, you will need to obtain permission directly from the copyright holder. To view a copy of this license, visit http://creativecommons.org/licenses/by/4.0/.

(c) The Author(s) 2019 